\title{
VERSIONES DEL ESTRECHO DE MAGALLANES. \\ EL PASO INTEROCEÁNICO DESDE LA PRIMERA CIRCUNNAVEGACIÓN DEL MUNDO HASTA LA CONQUISTA DEL REINO DE CHILE (1520-1552)
}

EZEQUIEL PÉREZ

\section{RESUMEN}

El desarrollo de la "conciencia planetaria" tiene como uno de sus epicentros el descubrimiento del paso interoceánico (1519-1522). El estrecho de Magallanes se convierte en un territorio multifacético ya que varía según la funcionalidad que le otorgan las fuentes. Nos proponemos analizar este espacio, atendiendo a los escritos de quienes formaron parte de la primera expedición que circunnavegó el globo y, en una segunda etapa, las cartas del conquistador de Chile, Pedro de Valdivia, quien incorpora el Estrecho a su proyecto territorial. La delimitación de los matices en la representación del paso interoceánico nos permite abordar el proceso de mundialización que se construye en la tensión entre los poderes coloniales de las diferentes regiones del Nuevo Mundo y las conexiones planetarias a las que están sometidos estos territorios. Chile.

PALABRAS CLAVE: estrecho de Magallanes, colonial, mundialización, territorio, conquista de VERSIONS OF THE STRAIT OF MAGELLAN.

THE INTEROCEANIC PASSAGE FROM THE FIRST CIRCUMNAVIGATION OF THE WORLD TO THE CONQUEST OF THE KINGDOM OF CHILE (15191520-1552)

\begin{abstract}
The development of "planetary consciousness" has as one of its epicenters the discovery of the interoceanic passage (1519-1522). The Strait of Magellan becomes a multi-faceted territory since its description varies according to the functionality that the colonial sources give it. We propose to analyze this space in the writings of those who participated in the first circumnavigation of the world and the letters of Pedro de Valdivia, the conquistador of Chile, who incorporates the Strait to his territorial project. The analysis of the representations of the interoceanic passage, allows us to approach the process of mundialization that is built on the tension between the local power in the New World and the planetary connections to which these territories are subjected.
\end{abstract}

KEY WORDS: Strait of Magellan, colonial, mundialization, territory, conquest of Chile. 


\section{INTRODUCCIÓN}

Uno de los episodios más relevantes para el desarrollo del proceso de mundialización, consecuente con el proyecto imperial español, es la primera circunnavegación al globo emprendida por Fernando de Magallanes el 20 de septiembre de 1519. El regreso de la nave Victoria en 1522 abrirá la posibilidad de que Occidente y Oriente se encuentren, por primera vez, en contacto. Se establece, entre estos dos puntos, una línea que reconfigura el orbe al sugerir la continuidad entre dos porciones del planeta que parecían imposibles de unir debido a la existencia de la masa territorial del Nuevo Mundo que las separaba y a un vasto océano de dimensiones desconocidas ${ }^{1}$.

La literatura de viajes contribuyó a acelerar el surgimiento de lo que Mary Louise Pratt llama -en su clásico Ojos imperiales. Literatura de viajes y transculturación- las "zonas de contacto" entre culturas diferentes ${ }^{2}$. Territorios que hasta ese momento se concebían de manera aislada, comenzaron a interactuar, de modo tal que las diversas formas de entender el espacio $y$ el tiempo confluyeron en un entramado de relaciones signadas por la disparidad de poder

1 Es cierto que los viajes de Marco Polo y El libro de las maravillas de John Mandeville permitieron un primer encuentro con el imaginario de Oriente e influenciaron a aquellos que dieron cuenta de su experiencia en el Nuevo Mundo. Si bien la historia de estas influencias excede los limites de este trabajo, siguen siendo fundamentales, entre otros, los análisis de Pastor, 2008. El segundo descubrimiento. La Conquista de América narrada por sus coetáneos (1492-1589). Edhasa, Buenos Aires; Rabasa, 2009. De la invención de América. La historiografía española y la formación del eurocentrismo. Universidad Iberoamericana, México; y Greenblatt, 2008. Maravillosas posesiones. El asombro ante el Nuevo Mundo. Marbot ediciones, Barcelona.

2 Debemos precisar, sin embargo, que Pratt remite en su análisis a los siglos XVII, XVIII y XIX, mientras que nuestro trabajo se centra en la primera mitad del siglo XVI. Si bien los contextos son diferentes, nos permitimos utilizar el concepto de "zona de contacto" en sintonía con la idea de "frontera colonial", tal y como lo entiende la propia autora: Pratt, 2010. Ojos imperiales. Literatura de viajes y transculturación. Fondo de Cultura Económica, Buenos Aires p. 34. Una visión alternativa e interesante es la que desarrolla Pamela Long bajo el concepto trading zones para aplicarlo a la temprana modernidad: I refer to the development of arenas in wich artisans and other entre los agentes involucrados: individuos que estuvieron separados por la geografía y la historia ahora coexisten en un punto, el punto en que sus respectivas trayectorias se cruzan ${ }^{3}$. Lugares de intercambio y negociación, espacios que se representan desde el "asombro" de los conquistadores $^{4}$, o que ponen en funcionamiento una imperturbable "lógica de colonización" de la otredad" ${ }^{5}$ en definitiva, el período que coincide con el surgimiento de las múltiples "historias conectadas" moldea un nuevo diagrama del orbe ${ }^{6}$.

¿Cuál es la forma en que se representa, para los receptores que distan del lugar en que se experimenta esta novedad, aquel territorio ubicado en los confines del mundo? En este trabajo abordaremos algunos de los documentos más significativos de los primeros años posteriores al descubrimiento del paso interoceánico, con el fin de dar respuesta a esta problemática. En primer lugar, partiremos del análisis de los navegantes que formaron parte de la expedición de Magallanes y protagonizaron ese magnífico "evento-mundo"7 en los albores de la modernidad, contribuyendo a moldear la concepción esférica y homogénea del planeta. Este cambio de paradigma en la cosmovisión

practitioners (trained as apprentices in workshops or in hands-on instruction at, say, construction sites) and learned men (trained in Latin at universities and other institutions) engaged in substantive communication and shared their respective expertise. Long, 2015. Trading Zones in early Modern Europe. Isis, 106(4), pp. 840-847. p. 842

Pratt, 2010, op. cit., p. 34.

Greenblatt, 2008, op. cit.

5 Subirats, 1994. El continente vacío. La conquista del Nuevo Mundo y la conciencia moderna. Siglo XXI, México, p. 60.

6 Gruzinski, 2010. Las cuatro partes del mundo. Historia de una mundialización. Fondo de Cultura Económica, México, p. 44. Gruzinski toma el concepto de connected histories del historiador Sanjay Subrahmanyam y advierte: La exhumación de dichas 'conexiones' históricas puede servir para desbaratar las aproximaciones o los a priori de la historia comparada, pero obliga también a reconocer que las historias son múltiples, incluso si, con frecuencia, se comunican entre sí o se relacionan en parte. Gruzinski, 2010, op. cit., p. 44.

7 Giucci, 2014. Tierra del Fuego: la creación del fin del mundo. Fondo de Cultura Económica, Buenos Aires, pp. 36-43. 
occidental estuvo complementado por numerosas experiencias que enfrentaron a los españoles con la otredad y forzaron a desarrollar un aparato conceptual cuya finalidad residía en aprehender las nuevas vivencias y darlas a conocer al público europeo ${ }^{8}$.

En segundo lugar, intentaremos delinear la forma en que el Estrecho fue descrito por quienes llevaron a cabo la conquista $y$ colonización del reino de Chile, adoptando una "visión periférica" "que complejiza la percepción del espacio en cuestión. Nos centraremos en las cartas del primer conquistador de la región, Pedro de Valdivia, y en la doble valencia del Estrecho durante su gobernación: por un lado, atenderemos a la importancia estratégica que tiene el paso interoceánico en el proyecto valdiviano $y$, por el otro, la peligrosidad de ser una frontera permeable a la entrada de extranjeros y españoles que disputen su poderío en la región. Así, nuestro objetivo es dar cuenta de la forma en que las representaciones del

8 Cf., Onetto, 2017a. Modernidad, historicidad y construcción de territorialidades desde un pasaje-mundo. El estrecho de Magallanes tras su 'descubrimiento'. Magallania, 45(2), pp. 37-58.

9 Tomamos el concepto de visión periférica del estudio de Beauchesne, 2013. Visión periférica. Marginalidad y colonialidad en las crónicas de América Latina (siglos XVI-XVII y XX-XXI). Iberoamericana, Madrid. Nos parece acertada la distinción entre los centros virreinales durante el período colonial y aquellos desprendimientos territoriales -tal es el caso de Chile- que no han sido objeto de interés para la Corona. Para Beauchesne, p. 16, el discurso periférico aunque mantiene vínculos sólidos con el discurso canónico (como el de Hernán Cortés, entre otros), enfatiza algunos mecanismos discursivos $y$ estrategias narrativas para retratar el medio físico y humano. En este trabajo nos interesa, sobre todo, plantear la cuestión de la legitimidad del enunciador en el débil entramado institucional del reino de Chile. Por último, el concepto de periferia es central tal y como lo entiende la autora canadiense: Empleo el término periferia a lo largo del libro porque estos territorios fueron descuidados [...] por el aparato colonial y percibidos como casi vacíos, p. 16.

10 Según Giucci, 2014, op. cit., p. 14, en su estudio sobre la "creación" del fin del mundo: El acontecimiento magnífico del siglo XVI fue en ese sentido la primera circunnavegación del planeta (1519-1522), que diversos cronistas definieron como un aporte sin precedentes a la verdad y al conocimiento. Con ese evento-mundo emerge una orgullosa conciencia de la ruptura histórica que
Estrecho varían a lo largo de la primera mitad del siglo XVI, teniendo en cuenta el punto de vista de los enunciadores y las circunstancias políticas, sociales y económicas que afectan los intereses de quienes enuncian. El estrecho de Magallanes, de esta manera, es un escenario propicio para analizar los matices que dieron impulso al surgimiento de una nueva imagen -e imaginación- del orbe.

\section{ESPACIALIZACIÓN DEL ESTRECHO DE MAGALLANES Y CONCIENCIA PLANETARIA: TESTIMONIOS DE LA PRIMERA CIRCUNNAVEGACIÓN}

La expedición encomendada a Fernando de Magallanes y Ruy Falero en 1519 marca el comienzo de una nueva "conciencia planetaria" en Occidente ${ }^{10}$. La empresa tenía un claro objetivo comercial: desembarcar en las islas Molucas en busca de la especiería que permitiera a España acceder a esos bienes sin transgredir

anticipa la eliminación de lo oculto. Al mismo tiempo, no podemos ignorar que el proceso de mundialización (Cf. Gruzinski, 2010, op. cit.) surge como corolario de un sistema político, económico y social en período de transformación. Es interesante retomar el clásico análisis de Perry Anderson en Lineages of the Absolutist State ya que permite caracterizar la transición que opera en el transcurso de los siglos XV y XVI hacia el estado absolutista, en donde la casa de los Habsburgo tuvo una influencia determinante a partir de su proyecto imperial: The most spectacular development of Charles V's reign was, of course, its vast enlargement of the Habsburg international orbit. In Europe, the Netherlands, the Franche-Comté and Milan were now added to the personal patrimony of the rulers of Spain, while Mexico and Peru were conquered in the Americas. During the life-time of the Emperor himself, the whole of Germany was a major theatre of operations over and above this hereditary possessions. Anderson, 1974. Lineages of the Absolutist State. Verso, London-New York, p. 68. Mauricio Onetto, al analizar el impacto de la primera circunnavegación a través del texto de Maximiliano Transilvano, acusa un cambio de época que deriva en lo que denomina "consciencia moderna": Las palabras del secretario de Estado son una declaración de que una nueva era se había instalado y nos advierten la relación que se puede establecer entre su aparición y el descubrimiento del Estrecho. Onetto, 2017a, op. cit., p. 42. Asimismo, Onetto entiende la Modernidad como una apertura temporal hacia lo nuevo y como una época que trajo consigo un nuevo principio rector: la posesión y aprehensión del mundo por la imagen. 
el tratado de Tordesillas ${ }^{11}$. Magallanes resulta ser el hombre adecuado para dar comienzo a aquella travesía, tanto por sus conocimientos en la navegación como por la información que poseía de fuente lusitana acerca de las especias que podían encontrarse en las Molucas ${ }^{12}$. Sin embargo, aquella finalidad principal por la cual se organiza la expedición tiene un estadio intermedio fundamental para asegurar que la circunnavegación del globo pudiera repetirse en futuros viajes: hallar el punto preciso del mapa en el que las embarcaciones dieran con el Mar del Sur desde Occidente.

La búsqueda del paso interoceánico ha sido documentada en varios escritos producidos en los años subsiguientes al retorno de la nave Victoria en 1522. Algunos de estos testimonios fueron esbozados por los mismos expedicionarios que sobrevivieron al viaje $y$ dieron cuenta de las dificultades y padecimientos que tuvieron que enfrentar; tal es el caso, por ejemplo, del

11 En Historia del estrecho de Magallanes, Martinic considera que Magallanes fundó su tesis principal en argumentos sólidamente construidos desde el conocimiento náutico y cosmográfico. Sostiene que tenía como objetivo alcanzar las Molucas sin violar los tratados entre Portugal y España: Ante el rey y sus consejeros, Magallanes defendió con calor su tesis de la navegación hacia las Molucas por la vía del occidente, más corta que la de los portugueses y en jurisdicción de Su Majestad, lo que le podría significar el dominio de las islas por cuanto -aseguró- caían dentro de los términos de la demarcación, con el consiguiente control absoluto del comercio de las especias. Martinic, 1977. Historia del estrecho de Magallanes. Editorial Andrés Bello, Santiago, p. 35.

12 Este conocimiento que poseía Magallanes no era indiferente a sus propios hombres, tal como lo expresa Antonio Pigafetta: Toda la tripulación estaba tan persuadida que este estrecho no tenía salida al oeste, que no se habría aun pensado en buscarla sin los grandes conocimientos del comandante en jefe. Este hombre, tan hábil como valeroso, sabía que era necesario pasar por un estrecho muy oculto, pero que él había visto figurado en un mapa que el rey de Portugal conservaba en su tesorería. Medina, 1888-1896. Colección de Documentos Inéditos para la Historia de Chile. Primera serie, Tomo II, p. 434. Queda en evidencia que Magallanes había accedido a información confidencial de la corona portuguesa y que utilizó ese conocimiento para diagramar el recorrido de su viaje hacia las Molucas.

13 La identidad de Francisco Albo ha sido puesta en duda por la crítica, llegando a considerar, incluso, que se trata de un alter ego de Sebastián Elcano. Vega, 2014. Los Andes $y$ el territorio de Chile en el siglo XVI. Descripción,
Derrotero de Francisco Albo ${ }^{13}$-escrito durante la navegación- y, principalmente, del célebre Viaje alrededor del mundo de Antonio Pigafetta ${ }^{14}$, cuya versión en francés debió aparecer por primera vez entre los años 1524 y $1525^{15}$. Lo curioso es que estos textos no surgen de una expedición que tenía como cometido la conquista y/o exploración de los territorios que visitaban a su paso ni la recopilación de información sobre las comunidades que se cruzaran en el trayecto, sino que son fruto de una empresa signada por la obtención inmediata de un rédito económico que podría virar las condiciones comerciales impuestas por el tratado entre la corona de Portugal y la de España ${ }^{16}$.

No es casual que entre los pedidos que el monarca elabora en las instrucciones previas a la partida de la flota, sobresalga aquel que obliga a los expedicionarios a que en ninguna manera no consintáis que se toque, ni descubra tierra, ni otra ninguna cosa dentro en los limites del serenísimo Rey de Portugal ${ }^{17}$. El mandato

reconocimiento e invención. Dirección de Bibliotecas, Archivos y Museos, Santiago, p. 152. Sin embargo, su presencia en la travesía puede constatarse en la Relación de la gente que va en las naos que su alteza manda enviar para el descubrimiento de la especiería de que va por capitán mayor Hernando de Magallanes (1519). Alli, Francisco Albo figura como contramaestre de la Trinidad, la nave que capitaneaba el mismo Magallanes. Medina, 1888-1896, op. cit., Primera serie, Tomo I, pp. 8-9.

14 Pigafetta, nacido en Vicenza y supernumerario de la expedición de Magallanes, escribió su Viaje al regresar en la nave Victoria y fue traducido al italiano entre los años 1534 y 1535 por Giovanni Battista Ramusio. Vega, 2014, op. cit., p. 150. Para un análisis de la influencia del Viaje de Pigafetta en sus contemporáneos y en la literatura latinoamericana, es fundamental el estudio de Robles, 2008. The First Voyage around the World: From Pigafetta to García Márquez. Guaraguao, 12(27), pp. 9-32.

15 Trabajaremos con la versión de Pigafetta que recoge Medina, 1888-1896, op. cit., Primera serie, Tomo II, que parte de una traducción al francés realizada por Carlos Amoretti en 1800 quien, a su vez, toma como fuente el manuscrito encontrado en la Biblioteca Ambrosiana de Milán.

16 Es ilustrativa la carta que Magallanes envía al rey Carlos V el 24 de octubre de 1518, en la cual el navegante remarca la importancia de prever el comercio con aquellos nativos que se encontrasen en el camino y de llenar las embarcaciones con las especias que esperaban hallar, augurando que la ganancia, placiendo a nuestro Señor, pueden ser veinte a uno. Medina, 1888-1896, op. cit., Primera serie, Tomo I, p. 18.

17 Medina, 1888-1896, op. cit., Primera serie, Tomo I, p. 58. 
proyecta un itinerario que no admite la desviación de la ruta trazada y que desalienta cualquier tipo de impulso exploratorio. En la misma línea, el rey deja en claro que la prioridad del recorrido es el mapeo de los puertos y la preparación de una ruta que facilite el comercio con las Molucas ${ }^{18}$. El trayecto que los españoles deben sortear no será más que un paso en los planes de la Corona: un espacio de tránsito que no tiene interés en sí mismo sino como un paraje donde las naves podrán abastecerse en futuras expediciones para acceder a Oriente.

El recorrido de las embarcaciones habilita, a la vez, el potencial flujo de los hombres entre dos puntos distantes. Este hecho hace que la travesía de Magallanes se convierta, antes de su partida, en una línea que conecta el lugar donde comienza el trayecto -San Lúcar de Barrameda- con un sitio de llegada incierto que cobija la proyección de la Corona sobre un territorio en el cual -según la previsión de sus gestores- nace la especiería.

En la misma instrucción, el asentamiento y población de ciudades españolas se vuelve un mandato en el momento en que los expedicionarios arriben al destino: E cuando con la buena ventura llegardes a las tierras e islas adonde hay las especierías, haréis asiento de paz e trata con el rey o señor de la tierra, como vieredes ques mas nuestro servicio e provecho ${ }^{19}$. El intercambio de bienes y de conocimientos es fruto de la circulación de los hombres que van en busca de un punto imaginario del mapa en que se localizarían las Molucas; la única premisa que guía a los tripulantes es la de avanzar hacia ese punto de llegada poco preciso que se halla del otro lado del orbe.

En sintesis, lo que estamos describiendo es un cambio en la apreciación del mundo que se traduce en una paulatina racionalización de las distancias y

18 El objetivo de la Corona queda expresado con claridad en la octava instrucción que esboza el rey antes de la partida de la flota magallánica, en la cual recomienda: Ternéis tal manera que de las cosas que llevais daréis al rey o Señor de la tierra algo en señal de amistad, para que quede con buena voluntad para cualquier navio que ahi llegare é toviere necesidad de agua, ó de los mantenimientos de la tierra, porque se los den con buena voluntad. Medina, 1888-1896, op. cit., Primera serie, Tomo I, p. 60.

19 Ibidem, pp. 61-62.

20 Para un estudio sobre el desarrollo científico en el siglo XVI en la representación de los puntos conectados por el desarrollo cartográfico sin precedentes en el siglo $\mathrm{XVI}^{20}$. Según Stephanie Massmann ${ }^{21}$, este viraje se da en el marco de un proceso de abstracción y homogeneización del espacio. En la medida en que estamos analizando un período de transición, podemos observar que este proceso no es uniforme sino que presenta varias contradicciones ya que:

[S]i bien [el navegante] utiliza $y$ colabora con la elaboración de una concepción abstracta del océano, por otra parte lo experimenta de una forma totalmente distinta, pues se somete a las vivencias de los tiempos del viaje, a las penalidades físicas que este implica y a los desafíos prácticos de la navegación ${ }^{22}$.

Esta aprehensión del espacio ligada a la navegación solo puede emerger en el marco del proyecto imperial que inaugura una nueva conciencia planetaria y se manifiesta de manera cabal en la expedición magallánica. Gruzinski (2010) sostiene que estas conexiones incentivadas por la corona española establecen acercamientos entre diferentes culturas y hacen convivir diversas formas de concebir el tiempo y el espacio:

El espacio de la monarquía se distingue ante todo por la circulación planetaria que se despliega en él y que, por la mezcla de hombres, de las sociedades y de las civilizaciones, lo irriga. En cualquier parte del mundo, el dominio ibérico aproxima, pone en relación o choca de frente con formas de gobierno, de explotación económica y de organización social. Súbitamente las temporalidades se empalman $[\ldots]^{23}$.

y la influencia del llamado "descubrimiento" de América en disciplinas tales como la geografía, la cartografía y las ciencias exactas, Cf. Trabulse, 1994. Ciencia y tecnología en el Nuevo Mundo. Fondo de Cultura Económica, México.

21 Massmann, 2013. Buscando camino por la mar: experiencia, geografía e imaginarios marítimos en relatos de navegación. Revista de Crítica Latinoamericana, 39(78), pp. 209-232, p. 216.

22 Ibidem, p. 217.

23 Gruzinski, 2010, op. cit., p. 47. 
Ahora bien, detengámonos en este lugar de tránsito que funciona, a su vez, como nudo que enlaza el comienzo y el fin del viaje, convirtiéndose en un medio para acceder a la tierra de la especiería. ¿De qué manera se representa el paso interoceánico en ese primer viaje alrededor del mundo? ¿Qué tipo de experiencia se manifiesta en los documentos? Como señalamos anteriormente, uno de los primeros testimonios que debemos revisar es el que se encuentra en el Derrotero escrito por Francisco Albo; se trata de un texto en el que se asientan diversas anotaciones sobre la altura de la derrota realizada por uno de los dieciocho sobrevivientes de la Victoria, la única de las cinco naves que retorna a Sevilla en 1522 habiendo circunnavegado el mundo ${ }^{24}$.

En las jornadas del Derrotero correspondientes al pasaje del Estrecho, la descripción de Albo está centrada, de manera enfática, en las dificultades que deben sortearse para atravesar el paso interoceánico por vía marítima. Es decir, abundan los consejos que el autor facilita para aquellos viajeros que intenten repetir la hazaña: y como vos emboquéis el estrecho, iros al sudueste por media canal; y como vos emboqueis, guardaos de unas bajas antes tres leguas de la boca, y después dellas hallareis dos isletas de arena ${ }^{25}$. Es evidente el desinterés de Francisco Albo por la tierra que queda al paso, el cual se manifiesta en la falta de profundidad acerca de lo que puede llegar a encontrarse en ella; sí se explaya, en cambio, en la descripción física del Estrecho: hay muchos ancones, y las sierras son muy altas y nevadas, y con muchos alboledo ${ }^{26}$.

24 Además de la nave Victoria y de la Trinidad, las otras tres embarcaciones que completaban la flota fueron las naves San Antonio, Concepción y Santiago.

25 Medina, 1888-1896, op. cit., Primera serie, Tomo I, p. 221.

26 Idem.

27 Como bien señala Emanuelle Vagnon, esta rectificación del saber de los antiguos tiene sus particularidades: la lettre de Maximilianus Transylvanus entretient justement un rapport ambigu à ce savoir car, tout en saluant l'expérience nouvelle des navigateurs et la correction des erreurs du passé, il reconnaît par ailleurs que ces témoignages viennent au contraire valider certaines de ces antiques connaissances. Vagnon, 2010. De la grèce antique au voyage de Magellan. Les modèles humanistes d'Antonio Pigafetta et de Maximilianus Transylvanus. Médiévales, 58, pp. 99-111, p. 109.
La mirada de Albo se posa sobre el espacio que transitan las embarcaciones y el paso interoceánico se configura, en esta primera aproximación, como un lugar peligroso en el que se debe maniobrar rápidamente, y con los recaudos adecuados, para alcanzar el Mar del Sur.

Así como -utilizando la terminología de Gruzinski- el descubrimiento del Estrecho irriga el conocimiento del mundo y posibilita la conexión entre espacios disímiles, al mismo tiempo se construyen esos lugares de tránsito que quedan fuera de los objetivos comerciales y, por lo tanto, en los márgenes de la representación del planeta. En este sentido, es indudable que la relación de Maximiliano Transilvano, escrita en 1522, sigue la misma línea que las instrucciones de la Corona y el Derrotero de Albo al proporcionar una explicación económica a la aventura de los expedicionarios. Sin embargo, Transilvano va un paso más allá al sugerir que el viaje de Magallanes implica una rectificación de aquellos autores antiguos que habían errado en la descripción del mundo ${ }^{27}$; es decir, estamos ante una re-significación de la ecúmene ${ }^{28}$ : antes tenemos agora conoscimiento, $y$ de cierto creemos ser fabulosas y cosas no verdaderas la que los autores antiguos dejaron escriptas; y que con la experiencia de los presentes pueden aquellas ser reprobadas ${ }^{29}$. Al mismo tiempo que el mundo se abre al conocimiento de los hombres, la experiencia de los viajantes desmitifica el espacio americano.

En el pasaje en el que Transilvano narra el cruce del Estrecho, conviven la experiencia de la aventura y el testimonio de los padecimientos que

28 Para Miguel Guerin los textos de viajes del Nuevo Mundo redefinen el espacio de la ecúmene, concepto que entiende no como la totalidad de las tierras habitadas sino como la totalidad de las tierras habitadas a que una cultura se siente referida. Guerin, 1992. El relato de viaje americano y la redefinición sociocultural de la ecúmene europea. Dispositio, 17(42-43), pp. 1-19, p. 6. Por esta razón el relato de viaje compite por la redefinición de la ecúmene y es doble ya que apela al espacio referido (los otros) y al punto de comparación (nosotros): El texto de un viajero no relata la transgresión de un límite, crea con su discurso, el limite entre dos lugares de la ecúmene, mediante lo cual explora y analiza los limites que definen la propia cultura, el centro de la ecúmene. Guerin, 1992, op. cit., p. 6.

29 Medina, 1888-1896, op. cit., Primera serie, Tomo I, p. 259. 
deben enfrentar los españoles para alcanzar los confines del mundo. El derrotero que lleva hacia la boca del Estrecho muestra la singladura de un clima hostil para los navegantes, en donde se consigna que más adelante pasaban, más frío les hacía, sin esperanza de hallar fin a aquella tierra firme, ni el pasage (sic) que para el otro mar buscaban ${ }^{30}$.

El hambre y el frío, las condiciones adversas de la navegación y el acento que se pone, desde el comienzo, en las dificultades de la travesía, dan cuenta de un espacio hostil para el proyecto de la expansión colonial en la región:

Procediendo pues por el estrecho, tardaron hasta pasar de la otra parte $y$ llegar al mar del sur, veinte y dos dias, en el cual tiempo jamas pudieron ver por ninguna de aquellas costas hombre alguno mortal, salvo que alguna noche vieron gran multitud de fuego en la tierra que estaba a la mano siniestra del estrecho hacia el austro, de donde congeturaron que habian sido vistos de los habitadores de aquella región y que se hacian aquellas almenaras de fuego unos a otros; nunca empero pudieron ver persona alguna. E como el capitán Magallanes considerase que aquella tierra era muy fragosa y que aun en aquel tiempo que duraban los días diez y nueves horas, hacía por alli grandísimos fríos y que era tierra de continuas y perpetuas frialdades en todos los tiempos del año, pareciole que era tiempo perdido haber de explorar ni saber lo que en tal tierra habia ${ }^{31}$.

La fragosidad de la tierra no deja de recordarle al lector que el viaje alrededor del mundo exige

30 Ibidem, p. 270.

31 Ibidem, p. 276.

32 Alejandra Vega nota, sin embargo, que no hay mención en Maximiliano Transilvano de la cadena montañosa que se vislumbra en el Estrecho y que ha sido asociada, desde los primeros momentos, con la hostilidad climática que describe el propio Transilvano en su texto. Vega, 2014, op. cit., p. 153. Para un estudio sobre el impacto de las primeras descripciones del Estrecho en la construcción de la "mala fama" del reino de Chile, cf. Onetto, $2017 \mathrm{~b}$. Reconsideraciones sobre la 'mala fama' de Chile durante el siglo XVI. Sophia Austral [online], 20, pp. 5-29.

sus sacrificios y que el tránsito incluye ese pasaje en el que se ponen a prueba los conocimientos marítimos de quienes dirigen la flota ${ }^{32}$. Puede sernos de utilidad, para entender la representación del Estrecho en estos discursos, la distinción que establece Michel de Certeau entre "lugar" y "espacio". El lugar es el orden (cualquiera que sea) según el cual los elementos se distribuyen en relaciones de coexistencia mientras que hay espacio en cuanto que se toman en consideración los vectores de dirección, las cantidades de velocidad y la variable del tiempo ${ }^{33}$. En esta línea de análisis, tanto Francisco Albo como Maximiliano Transilvano convierten en espacio el trayecto que une el punto de partida -San Lúcar de Barrameday el arribo a las Molucas, otorgando a esos sitios antes estáticos del mapa las conexiones que lo convierten en un "lugar practicado" 34 . El Estrecho queda inmerso en esa configuración como un lugar que atenta contra la espacialidad del recorrido.

El Viaje alrededor del mundo, de Antonio Pigafetta, supondrá un viraje significativo respecto a la representación de la travesía magallánica, tanto por la forma discursiva en que aborda la narración del viaje ${ }^{35}$ como por la meticulosidad con que describe el paso interoceánico:

La costa de este estrecho que del lado izquierdo se dirige al sudeste, es baja: dimosle el nombre de Estrecho de los Patagones. A cada media legua se encuentra en él puerto seguro, agua excelente, madera de cedro, sardinas y mariscos en gran abundancia. Había también yerbas $y$, aunque algunas eran amargas, otras eran buenas para comer, sobre todo una especie de apio dulce que crece en la vecindad de las fuentes y del Artes de hacer. Universidad Iberoamericana, México, p. 129.

34 Idem.

35 Emanuelle Vagnon considera que L-hypothèse la plus probable est donc que Pigafetta suivat surtout un modèle éditorial, de difusion de ce genre de littérature, destiné à un certain public. La forme comptait autant que le fond quand il s'agissait de plaire à un milieu littéraire avide de récits de voyage et friand de beaux manuscrits cartographiques richement décorés. Vagnon, 2010, op. cit., pp. 107-108. 
cual nos alimentamos a falta de otra cosa mejor: en fin, creo que no hay en el mundo un estrecho mejor que éste ${ }^{36}$.

La perspectiva espacial de Pigafetta hace hincapié en la viabilidad de la posesión territorial. La insistencia en el nombramiento del Estrecho pone de relevancia el interés de poseer aquella tierra ubicada en el extremo sur del Nuevo Mundo. El catálogo de alimentos y la presencia del agua que pueden abastecer a las embarcaciones que hagan puerto, dan cuenta de una descripción que se aparta de la observación lejana del navegante absorto en el tránsito y enfatiza la potencialidad de una tierra todavía inexplorada. También abunda en observaciones proto-etnográficas que son producto de su curiosidad por el Otro, a diferencia de la Relación de Transilvano y, por supuesto, del Derrotero de Albo.

Sin embargo, la imposibilidad de repetir el trayecto se halla en la travesía misma; poco después de abandonar el Estrecho y seguir rumbo a las Molucas, Pigafetta anota: No pienso que nadie en el porvenir ha de querer emprender semejante viaje ${ }^{37}$. Esta aparente contradicción es sugestiva ya que nos habla de una tensión que atraviesa el relato de Pigafetta. Para Guillermo Giucci, el descubrimiento del estrecho patagónico es el punto inicial de la conciencia planetaria, es decir, es el puente hacia el gran mundo, hacia lo redondo, hacia la totalidad ${ }^{38}$; al mismo tiempo, sostiene que en los relatos de testigos y cronistas quinientistas, el estrecho de Magallanes aparece como periferia, lugar de tránsito, pasaje hacia $^{39}$. Lo curioso es que en la descripción de Pigafetta parece ocurrir lo contrario: el estrecho de Magallanes surge como un territorio habitable que ocupa un lugar central en el derrotero, mientras que la travesía se convierte en un suceso irrepetible. Esta observación nos permite sospechar que la gestación

36 Medina, 1888-1896, op. cit., Primera serie, Tomo II, p. 437.

37 Ibidem, p. 439.

38 Giucci, 2014, op. cit., p. 33

39 Ibidem, p. 34.

40 Medina, 1888-1896, op. cit., Primera serie, Tomo IV, pp. 224-225

41 El señalamiento de Valdivia acerca de las condiciones en que había dejado desamparada la tierra Diego de Almagro aparece en las primeras cartas enviadas desde el reino de Chile: Sepa Vuestra Majestad que cuando el Marqués don Francisco Pizarro me dio esta empresa, no había de una conciencia planetaria en Occidente no siempre trajo consigo la potencia de una comunicación fluida con la otredad, sino que presentó -como en el caso de Pigafetta- asimilaciones diversas.

\section{EL ESTRECHO DE MAGALLANES DURANTE LOS PRIMEROS AÑOS DE LA GOBERNACIÓN DE PEDRO DE VALDIVIA}

Apenas una década después de la primera circunnavegación del globo, el estrecho de Magallanes adquiere nuevos visos en el proyecto de la corona española. ¿Qué sucede en el reino de Chile? ¿Qué lugar ocupa el Estrecho en la configuración territorial de ese espacio? En términos jurídicos, la capitulación de Toledo de 1534 había delimitado de manera precisa -y también conflictiva- las fronteras entre la gobernación de Francisco Pizarro y Diego de Almagro. A este último se le daba permiso para conquistar, pacificar y poblar las provincias y tierras que hubiere en las dichas doscientas leguas que comiencen desde donde se acaban los limites de la gobernación [del] capitán Francisco Pizarro al levante que es hacia el estrecho de Magallanes ${ }^{40}$. La incursión de Diego de Almagro en 1535 al reino de Chile fracasa en apenas unos meses de campaña y sus hombres no logran avanzar más allá del valle de Aconcagua, razón por la cual el conquistador decide volver sobre sus pasos y retornar al Perú en 1537. La re-significación del territorio correspondiente al estrecho de Magallanes tiene uno de sus epicentros en las cartas del conquistador extremeño Pedro de Valdivia, quien vuelve a posar sus intereses sobre la tierra abandonada por Almagro. La concepción espacial valdiviana traza una línea donde el reino de Chile se erige en la tensión provocada por la infamia que ha impreso Diego de Almagro en el territorio y su propia actuación que busca des-infamar la tierra ${ }^{41}$.

hombre que quisiese venir a esta tierra, y los que más huían de ella eran los que trujo el Adelantado don Diego de Almagro, que, como la desamparó, quedó tan mal infamada que como de la pestilencia huían de ella. Valdivia, 1986 [1545]. Cartas de relación de la conquista de Chile, Edición de M. Ferreccio Podestá, Editorial Universitaria, Santiago, p. 27. Lucía Invernizzi considera que la tarea de Valdivia en sus cartas de relación es convertir en deseable un espacio in-deseable para la corona española. Invernizzi, 1984. La representación de la tierra de Chile en cinco textos de los siglos XVI y XVII. Revista Chilena de Literatura, 23, pp. 5-37, p. 9. 
Lo cierto es que una de las preocupaciones centrales de Pedro de Valdivia durante los primeros años de la conquista de Chile será la irregularidad en el nombramiento de su cargo. Esta es una de las razones por las cuales, en las primeras cartas conservadas del conquistador extremeño, la insistencia en la política espacial que privilegia la fundación y el sostenimiento de ciudades se condice con el pedido reiterado al monarca de otorgarle el título ansiado que despeje cualquier tipo de duda sobre la legitimidad de su empresa ${ }^{42}$. El temor de Valdivia se asienta en que algún otro español arribe al reino de Chile con la intención de disputarle el poder, lo que repercutiría en un gran perjuicio para la región ${ }^{43}$. Lo expresa con claridad en la carta que envía a Carlos V en 1545 desde la ciudad de La Serena: El peso de esta tierra y de su sustentación y perpetuidad $y$ descubrimiento, y los mesmo de la de adelante, está en que en estos cinco o seis años no venga a ella de España por el Estrecho de Magallanes capitán proveído por vuestra Majestad, ni de las provincias del Perú, que me perturbe ${ }^{44}$.

Si bien el estrecho de Magallanes aparece -al igual que en las experiencias precedentes- como un límite que da forma al espacio de la conquista, en el

42 La legitimidad del nombramiento de Valdivia era en extremo cuestionable, incluso si consideramos el título de teniente de gobernador. Según Valdivia, el nombramiento inicial de 1539 remitía a una cédula firmada en Monzón por Carlos V, en 1537, en la que permitía a Pizarro -en caso de que Almagro estuviese muerto- enviase a poblar e conquistar e gobernar el Nuevo Toledo e las provincias de Chili. Valdivia, 1986 [1550], op. cit., p. 117. Sergio Correa Bello señala que en ninguno de los documentos se encuentran las provincias de Chile dentro de los territorios atribuidos a Pizarro, sino que se repite la misma capitulación entregada a Diego de Almagro. Valdivia, por su parte, no ignoraba este exceso en las atribuciones de Pizarro: Asi se explica que el conquistador tomara posesión de la tierra en nombre del Rey y no de Pizarro. Correa, 1986. Los fundamentos legales del primer nombramiento de Pedro de Valdivia. Historia, 21, pp. 167-176, p. 176.

43 Las cartas valdivianas tienen una fuerte construcción retórica que ha sido analizada por Cedomil Goic. Según el crítico chileno, la virtud que emana de estas cartas como la principal característica del conquistador es la prudentia. Ella encamina la voluntad del conquistador de persistir con calculados pasos, en medio de los trabajos y dificultades, los gastos y las deudas, que traen consigo el descubrir, conquistar y poblar. Goic, 2006. Letras del reino de Chile. Iberoamericana Vervuert, Madrid, p. 48.

44 Valdivia, 1986 [1545], op. cit., p. 47. caso de Valdivia se trata de una frontera que se puede franquear y que, a causa de esta misma permeabilidad, se convierte en una amenaza para quienes están llevando a cabo el descubrimiento y poblamiento del reino de Chile. Al mismo tiempo, la naturaleza de esta contingencia es peculiar: el Estrecho no es solo un punto de conexión entre diversos lugares sino que, además, es una vía de acceso al Nuevo Mundo. El peligro acecha en aquellos extranjeros que puedan instalarse en el territorio y dificulten la tarea de los españoles; simultáneamente, según la perspectiva valdiviana, los mismos españoles pueden desestabilizar el reino de Chile. En el extremo sur, en una zona poco explorada y casi desconocida, el estrecho de Magallanes es el andamiaje sobre el que se erigen los temores del gobernador.

Como consecuencia de esta situación, Valdivia opone a la inestabilidad de su cargo una fuerte política espacial basada en la fundación de ciudades en la zona central del reino de Chile ${ }^{45}$. La primera de las ciudades que funda es Santiago del Nuevo Extremo en 1541 mientras ordenaba levantar, hacia el norte, la ciudad portuaria de La Serena; más tarde, como él mismo lo expresa al emperador Carlos V en la última de sus cartas conservadas ${ }^{46}$, en

45 Para Mateo Martinic, el centralismo es una de las causas por las cuales ni siquiera la historiografía chilena dio cuenta de la importancia de la primera circunnavegación en la construcción territorial de Chile. Martinic considera la trascendencia de la empresa magallánica por tres razones: en primer lugar porque con ese acontecimiento Chile ingresa a la Geografía Universal; en segundo lugar porque con el mismo Chile se incorpora a la historia de los pueblos (comienza la Historia de Chile); y por último porque tienen principio la Historia Natural y Marítima chilenas, la Cartografía nacional y la Astronomía chilena. Martinic, 2016. Una travesía memorable. Hallazgo y navegación del Estrecho de Magallanes (21 octubre-28 noviembre 1520). Talleres La Prensa Austral, Punta Arenas, p. 197.

46 Se conservan once cartas escritas por Pedro de Valdivia con diferentes destinatarios $y$ en diversas circunstancias históricas, desde la carta de condolencia que envía a Gonzalo Pizarro el 20 de agosto de 1545 con motivo de la muerte de Francisco Pizarro, hasta la última que envía al emperador Carlos V desde Santiago el 26 de octubre de 1552. Para profundizar en las particularidades discursivas de las cartas valdivianas cf. Ferreccio, 1991. El epistolario cronístico valdiviano y el scriptorium de conquista. En Cartas de don Pedro de Valdivia: que tratan del descubrimiento y conquista de la Nueva Extremadura. Edición de M. Rojas-Mix. Transcripción y notas de M. Ferreccio Podestá, Lumen, Barcelona; Goic, 2006, op. cit. 
1550 fundó la ciudad de Concepción con cuarenta vecinos; en marzo de 1551 la ciudad de La Imperial con ochenta vecinos; en febrero de 1552 la ciudad de Valdivia donde tienen de comer cient vecinos ${ }^{47}$ $y$, en abril de ese mismo año, la ciudad de Villarrica con cincuenta vecinos.

La fundación y sustentación de ciudades es la estrategia que complementa las acciones de la guerra. Sin embargo, no deja de pensar en estos primeros años en la posibilidad de explorar el espacio desconocido que se extiende desde la última ciudad hasta el estrecho de Magallanes. En efecto, el 3 de septiembre de 1544, luego de cuatro años de estar asentado en el reino de Chile, Pedro de Valdivia otorga un poder al navegante Juan Bautista Pastene para descubrir la costa desde el puerto de Valparaíso hacia el sur. Pastene -un genovés que había llegado a Chile en 1544 y se había convertido en teniente de capitán general en el mar- es una pieza clave en la exploración marítima durante el período valdiviano; el mismo gobernador le otorga el poder mencionado por ser persona de prudencia y confianza y práctico en las cosas de la guerra, así con indios, como en nuevos descubrimientos ${ }^{48}$. El objetivo del mandato valdiviano consistía en que Pastene:

Salte en tierra todas las veces que le pareciere con la gente que fuere menester, por saberlo bien hacer, y me tome lengua en toda la costa desde el paraje deste puerto de Valparaíso, hasta el estrecho de Magallanes, y me descubra la costa $y$ puertos que hay en ella, y me traiga verdadera relación ${ }^{49}$.

47 Valdivia, 1992 [1552], op. cit., p. 177.

48 Medina, 1888-1896, op. cit., Primera serie, Tomo VIII, p. 72. El poder al que nos referimos se halla en la Colección de Documentos Inéditos para la Historia de Chile, y lleva el título de Poder que dio Pedro de Valdivia, gobernador de la Nueva Extremadura, a Juan Bautista Pastene, su teniente de capitán general en la mar, para el viaje a que le enviaba a descubrir la costa desde el puerto de Valparaíso hasta el estrecho de Magallanes; y a continuación la instrucción, y la relación del suceso del viaje desde 4 hasta 30 de septiembre de 1544. Medina, 1888-1896, op. cit., Primera serie, Tomo VIII, pp. 71-81.

Sobre la función de la escritura en la conquista y colonización
Rápidamente Valdivia intenta obtener información de esos territorios que quedan fuera de las representaciones cartográficas que circulaban en España y el Nuevo Mundo. Por esta razón, ordena explorar las costas de aquellas tierras en las que todavía no hay una clara delimitación por parte de la Corona. No es casual que la expedición que lidera Juan Bautista Pastene esté compuesta, entre otros, por Juan de Cárdenas, secretario personal de Pedro de Valdivia y hombre de confianza del gobernador, el cual tiene la tarea de dar testimonio por escrito de la tierra donde el dicho mi capitán saltare, y de la posesión que tomare della ${ }^{50}$. El objetivo del gobernador reside en extender el conocimiento de la región y, sobre todo, en tomar posesión de aquellos territorios que sus hombres logren explorar camino al Estrecho. La escritura funciona, en este caso, como una herramienta de legitimación que garantiza la posesión del territorio ${ }^{51}$.

Es cierto que, en esta instancia, Valdivia sigue concibiendo el espacio de su gobernación desde la precariedad institucional que la caracteriza. La fundación de ciudades y el levantamiento del cabildo que afirma, una y otra vez, la decisión de los vecinos de confiar en su persona la dirección militar y administrativa del reino de Chile, no hacen más que insistir en el pedido que estructura la mayoría de las cartas que el extremeño dirige al rey Carlos V, enfatizando los trabajos $y$ padecimientos ${ }^{52}$ que el gobernador debe soportar para sostener la integridad de las ciudades y de sus vecinos. El hambre, como consecuencia de la decisión de los indios de no producir alimentos para los españoles, se combate con las manos de

de América sigue siendo fundamental el aporte de Martin Lienhard, quien sostiene que la escritura, en este contexto, se manifiesta como una práctica política, religiosa y también jurídica. La función que prevalece es la performativa: la escritura se convierte en sinónimo de "hacer" y su palabra es ley. Lienhard, 1990. La voz y su huella: escritura y conflicto étnico-social en América Latina. Casa de las Américas, La Habana, p. 26.

52 Un estudio exhaustivo e iluminador sobre los "trabajos" realizados por los españoles en las cartas valdivianas se halla en Invernizzi, 1990. Los 'trabajos de la guerra' y 'los trabajos del hambre': dos ejes del discurso narrativo de la Conquista de Chile (Valdivia, Vivar, Góngora Marmolejo). Revista Chilena de Literatura, 36, pp. 7-15. 
los mismos conquistadores, quienes se encargan de la labranza y la sustentación de las sementeras para que el proyecto imperial triunfe más allá de los centros coloniales ${ }^{53}$.

Teniendo en cuenta los problemas internos que asedian a Valdivia en los primeros años de la conquista, podríamos sostener que el viaje que el gobernador encomienda a Pastene en 1544 es el preludio de una nueva configuración de las fronteras que enmarcan su poder. A la primera estrategia de fundación y asentamiento, Valdivia suma la exploración y el descubrimiento de los territorios que se encuentran por fuera de su jurisdicción e intenta darle un sustento institucional a partir de la tarea encomendada a su secretario Juan de Cárdenas ya que, como señalara Stephen Greenblatt, un escribano facilita una transacción con una forma de temporalidad más inmediatamente útil, la forma institucional asegurada por la escritura $^{54}$. La exploración de las costas del Mar del Sur tiene como finalidad la incorporación de un territorio que escapa al conocimiento occidental y se sume en un vacío representacional que el gobernador intenta remediar: entre Valparaíso $y$ el estrecho de Magallanes hay un espacio que debe ser llenado de escritura ya que no hay noticias certeras de lo que se encuentra en medio de esos dos puntos referenciales. Como señalamos antes, la exploración permitiría delinear esos bordes ausentes de los mapas y reconocer los puertos que no han sido plasmados por los cosmógrafos que ignoran la importancia del reino de Chile.

¿Qué concepción territorial sostiene esta primera parte de la gobernación de Valdivia? ¿Qué lugar tiene el estrecho de Magallanes en la representación del reino de Chile como un espacio asequible para la conquista? En este sentido, hemos comprobado una determinación notable en la inestabilidad institucional de la gobernación que da forma a la descripción del territorio. El estrecho de Magallanes cambia su carácter según el punto de vista desde el cual se lo perciba: para los navegantes que buscan la tierra de la especiería es un lugar de tránsito o, en el mejor de los casos,

53 Así lo expresa el mismo Valdivia en un pasaje de la carta enviada a Carlos V en 1545, en donde enfatiza el trabajo de los conquistadores en la sustentación del reino de Chile: los trabajos de la guerra, invictísimo César, puédenlos pasar los hombres, porque loor es al soldado morir peleando; un territorio en potencia. Para Pedro de Valdivia, que evidencia un afán de permanencia en el reino de Chile, es el punto de quiebre: el sitio que filtra los elementos que pueden llegar a corromper su poder. Como bien lo expresa el gobernador, las consecuencias de que ingresen españoles por el Estrecho pueden ser catastróficas para la región: $Y$ si nos viesen litigar sobre la tierra, está tan vedriosa que se quebraría y el juego no se podría tornar a entablar en la vida ${ }^{55}$. Estamos ante un caso particular del desarrollo de la conciencia global en Occidente ya que podríamos pensar a Pedro de Valdivia como un sujeto que busca cerrar las fronteras ante el peligro inminente de la desestabilización que representa para su proyecto la porosidad del paso interoceánico.

\section{LA INCORPORACIÓN DEL ESTRECHO AL PROYECTO TERRITORIAL VALDIVIANO}

Las dos embarcaciones comandadas por Juan Bautista Pastene no logran el cometido de alcanzar el estrecho de Magallanes, aunque sí recorren gran parte de las costas que se encuentran al sur de Valparaíso. En 1548, desde la ciudad de Los Reyes, Valdivia escribe al Consejo de Indias dando cuenta de los resultados de la expedición de su teniente de capitán general en el mar y plantea un interés inédito sobre aquel espacio que permanece como una incógnita para el conocimiento occidental:

Cuando envié a descubrir la costa, como a nuestro Monarca escrebí, y a tomar la posesión de la tierra en nombre de vuestra Alteza, llegó el navio que envié cerca del estrecho de Magallanes; y si vuestra Alteza es servido que el estrecho se navegue, me lo envie mandar, porque no está en más navegarse, mediante la voluntad de Dios, de ser vuestra Alteza dello servido; porque, aunque yo para ello me haya de empeñar en más de lo empeñado, por más servir a vuestra Alteza, haré de manera que desde el día

pero los de la hambre concurriendo con ellos, para los sufrir más que hombres han de ser. Valdivia, 1986 [1545], op. cit., p. 33.

54 Greenblatt, 2008, op. cit., p. 128.

55 Valdivia, 1986 [1545], op. cit., p. 47. 
que llegare el mandado de vuestra Alteza en muy breve haya nao en Sevilla que lo haya pasado ${ }^{56}$.

El Estrecho deviene en un espacio navegable y emerge la promesa de entablar un contacto fluido entre Sevilla y el reino de Chile que se podría concretizar tan rápido como el conquistador extremeño posea el permiso para explorar el lugar. Ahora bien, ¿cuál es la razón por la cual Valdivia deja de considerar el paso interoceánico como una amenaza para concebirlo como parte de un entramado planetario? La respuesta, por supuesto, no puede agotarse en la referencia histórica, pero podemos suponer que debe haber influido sobremanera el hecho de que en 1548 el presidente La Gasca -tras haber aplacado el levantamiento de Gonzalo Pizarro en Perú- otorgara a Pedro de Valdivia el título de gobernador del reino de Chile. En la misma carta destinada al Consejo de Indias, Valdivia comenta que La Gasca me proveyó en su real nombre de Gobernador y capitán general de aquella gobernación del Nuevo Estremo ${ }^{57}$. La frontera que escapaba de los límites institucionales de la gobernación y se abría como un campo de penetración de fuerzas extrañas a su poder durante los primeros años de la incursión española, se presenta, luego del nombramiento, como un territorio plausible de ser anexado.

La expedición trunca de Juan Bautista Pastene retorna con un conocimiento novedoso que contribuye a los planes de expansión de los dominios del gobernador. La operación que realiza Valdivia en sus cartas es la de producir una representación sobre aquel punto ciego del mapa ${ }^{58}$. Considerando que el territorio es un espacio en el que se ha proyectado trabajo, energía $e$ información y que, en consecuencia, revela relaciones marcadas por el poder ${ }^{59}$, se puede delimitar el accionar discursivo del gobernador de Chile respecto a quienes lo antecedieron: Valdivia

56 Ibidem, p. 80.

57 Ibidem, p. 78.

58 Lefebure sostiene que la representación del espacio es la que realizan, entre otros, los científicos, urbanistas y tecnócratas que conceptualizan el espacio: This is the dominant space in any society (or mode of production). Conceptions of space tend (...) towards a system of verbal (and therefore intellectually worked out) signs. territorializa el espacio correspondiente al estrecho de Magallanes como nunca antes había ocurrido.

Una primera observación se vuelve necesaria: para Valdivia, el sur exige su poblamiento para garantizar la estabilidad de todo el reino de Chile. Esa es su manera de proyectar las relaciones políticas sobre un territorio que intenta incorporar a sus planes primigenios. En 1550, cuando escriba a sus apoderados en la Corte para que den información al rey sobre su actuación en el Nuevo Mundo, Valdivia recomendará:

Informar así mismo cómo, desde a ocho o diez días, llegó a este puerto con la galera e navio el capitán e piloto Juan Batista Pastene. Luego le despaché a que corriese la costa de Arauco e trajese los navios cargados de comida, e hice pasar el río grande al capitán Jerónimo de Alderete con cincuenta de caballo, y se pasó muy bien, e que fuesen a correr Arauco e hacer espaldas a la armada, e asi se hizo. Vieron la más linda tierra del mundo todo, sana e apacible e sitio para poblar una cibdad mayor que Sevilla ${ }^{60}$.

Es interesante la comparación de esa tierra todavía despoblada de españoles -aunque no de representación- con la ciudad de Sevilla puesto que, como en reiteradas ocasiones, Valdivia está intentando mensurar ese sitio apartado de la mirada de la Corona y volverlo asequible para quienes tienen en sus manos el destino de su gobernación. Es probable que el objetivo fuera acercar esos dos espacios y establecer un lazo entre ellos, así como los navíos pudieran llegar a conectar el reino de Chile con la ciudad de Sevilla a través del Estrecho. A la vez, la proyección de Valdivia sobre los territorios del sur apela a la imagen de un lugar paradisíaco que vendría a paliar muchos de los inconvenientes que tuvieron que sortear los

Lefebvre, 1974. The production of space. Blackwell, Oxford-Cambridge, p. 39. Un análisis que complementa la perspectiva de Lefebvre sobre la producción del espacio es el que lleva a cabo Raffestin, 2011. Por una geografía del poder. El Colegio de Michoacán, México.

59 Raffestin, 2011, op. cit., p. 102.

60 Valdivia, 1986 [1550], op. cit., p. 109. 
españoles en las ciudades fundadas desde 1541.

La necesidad de avanzar sobre tierras que prometían no solo recursos auríferos, sino también gran cantidad de indios para entregar a los vecinos y combatir el hambre, hace que la preocupación de Valdivia por el sur de Chile se convierta en el tema más importante de la segunda etapa de la empresa. Gilberto Triviños considera que hay una autoficcionalización en las cartas valdivianas que remite a un tiempo heroico en donde el protagonista -en este caso, el mismo gobernador- se representa a sí mismo como un varón superior cuyo interés principal es servir a Dios y al Rey, no buscar oro ${ }^{61}$. Es por esta razón que, al gobernador ecuánime y buen vasallo, en esta etapa lo complementa el militar aguerrido que llevará a cabo la conquista de Arauco para reforzar la tierra que se halla entre el desierto de Atacama y el estrecho de Magallanes:

Emprenderé lo de adelante $y$ poblaré una cibdad donde comienza la grosedad de la gente y tierra, que yo la tengo bien vista, y en demanda desta noticia, ascuras y a la ventura han andado todos los españoles del Río de la Plata y los que han salido al Perú ahora de aquellas partes. Y yo espero en la buena de vuestra Majestad y con lumbre ir a cosa sabida y a la causa ${ }^{62}$.

Valdivia se declara poseedor de un saber que los demás conquistadores provenientes del Río de la Plata y del Perú desconocen. Ese saber está relacionado con la noticia de la tierra y el conocimiento del espacio que ilumina su proyecto. La metáfora de la luz y la oscuridad da comienzo, a la vez, al discurso de la guerra frente a un enemigo que se manifiesta implacable. Pero, simultáneamente, señala un vacío -que él mismo debe llenar- en la representación del espacio que está al sur del reino de Chile. El conocimiento del gobernador clarifica y delinea los contornos del

61 Triviños, 1996. El mito del tiempo de los héroes en Valdivia, Vivar y Ercilla. Revista Chilena de Literatura, 49, pp. 5-26, p. 7.

62 Valdivia, 1986 [1549], op. cit., p. 82.

63 Ibidem, pp. 160-161.

64 Valdivia insiste en el respeto que su gobernación tiene por el aspecto institucional, sobre todo, en las dos últimas territorio que quedaban fuera de la producción espacial de la Corona e, incluso, remienda muchos de los errores en los que habían caído los expedicionarios que le precedieron y que, confundidos, cartografiaron de manera imprecisa el estrecho de Magallanes:

[P]orque cuando envié al piloto Joán Bautista Pastene, mi teniente general en la mar, al descubrimiento de la costa hacia el Estrecho, rigiéndose por las cartas de marear que de España tenía imprimidas, hallándose en cuarenta e un grados estovo a punto de perderse; por do se ve que las cartas que se hacen en España están erradas en cuanto el Estrecho de Magallanes ${ }^{63}$.

Comienza a invocarse el espacio magallánico, antes lejano e inestable, como un punto en el que se aúnan los afanes imperiales de España con el ansia de legitimar y reforzar el gobierno de Valdivia. En otros términos, sostenemos que el gobernador de Chile se encarga de recalcar la confluencia de la concepción planetaria del proyecto imperial y de la estabilización que puede otorgar su perspectiva colonizadora si se le permitiese avanzar en la conquista del sur. En primer lugar, porque Valdivia reorganiza sus planes enfatizando el potencial resquebrajamiento del territorio debido a los puntos de ingreso que podrían poner en jaque el armado político que ha ido entretejiendo a lo largo de diez años. Por esta razón, una vez obtenido el título de gobernador del reino de Chile de manos del presidente La Gasca, Valdivia recuerda, una y otra vez, su desempeño como buen vasallo que cumple con todas las condiciones institucionales que la Corona exige a sus conquistadores: el requerimiento, el reparto de indios en encomienda controlado por el Cabildo y la justicia de un gobernador ecuánime que beneficia a los que se destacan en el servicio al rey ${ }^{64}$.

cartas destinadas al Príncipe Felipe y a Carlos V. Además de recalcar que presta especial atención al tratamiento de los indios, promueve la conversión a nuestra santa fee y creencia de los naturales. Valdivia, 1986 [1552], op. cit., p. 181. En la misma jerarquía se halla el acatamiento al pedido real de sobre los casados, instando a sus hombres a que vayan o envien por sus mujeres, Ibidem, p. 182. 
En segundo lugar, porque produce discursivamente un territorio que todavía no ha sido explorado e imagina el asentamiento de los españoles en un lugar que supone apto para conformar la mejor ciudad de todo el Nuevo Mundo. El sur es la nueva obsesión de Valdivia y, como corolario -no conforme con el repartimiento de tierras conferido por La Gasca para su gobernaciónsolicita al rey y a sus intermediarios en la Corte: $[\mathrm{H}]$ acerme merced de me alargar los limites della, que sean hasta el Estrecho dicho, la costa en la mano, e la tierra adentro hasta la Mar del Norte ${ }^{65}$. Hacia 1550, Valdivia pone en funcionamiento una maquinaria representacional que concibe al reino de Chile como una unidad, homogeneizando un espacio que había sido fragmentado por la ya mencionada capitulación de 1534: Y porque de alli al Estrecho de Magallanes es la tierra que puede haber poblada poca, y la persona a quien se diese antes estorbaría que serviría, e yo la voy toda poblando y repartiendo a los vasallos de vuestra Majestad y conquistadores ${ }^{66}$.

Poblar, repartir la tierra y consolidar su dominio: esa es la proyección del espacio que desarrolla Valdivia. Desde la primera carta de 1545 hasta 1550, el sur del reino de Chile adquiere múltiples facetas; lo cierto es que Valdivia cambia el modo de entender e imaginar ese territorio antes descartado por los españoles que habían realizado la primera vuelta al mundo en 1522: el estrecho de Magallanes es visto como un punto de llegada, el fin de una tarea que tiene como objetivo ir poblando y sustentando los territorios que vayan conquistando en el camino. Una vez consolidada la legitimidad anhelada en los primeros años, Valdivia se permite incorporar ese espacio en el proyecto imperial, es decir, evidenciar la importancia del estrecho de Magallanes en los planes que competen a la Corona:

[D]onde más su Majestad y vuestra Alteza el día de hoy pueden ser servidos es en que se navegue el Estrecho de Magallanes, por tres cabsas, dejadas las demás que se podían dar. La primera, porque toda esta tierra y la Mar del Sur la terná vuestra Alteza en España y ninguno se atreverá a hacer cosa que no deba; la segunda, que se terná muy a la mano toda la contratación de la especiería; y la tercera, porque se podrá descubrir e poblar esotra parte del Estrecho, que, según estoy informado, es tierra muy bien poblada ${ }^{67}$.

La importancia de su pedido, entonces, tiene varias aristas que atienden a diferentes estrategias de producción espacial. La primera de las causas que utiliza para justificar su posición sobre el Estrecho, apela a la soberanía de España en tierras alejadas de la metrópoli. Extender el dominio de la Corona, en lugares tan remotos como el estrecho de Magallanes, coincide con el fuerte anclaje territorial que fundamenta el proyecto imperial español. La segunda de las causas incorpora ese dominio a la escala planetaria ya que vuelve a adquirir la significación de ser el lazo que sirve para conectar a España con la tierra donde nace la especiería. Nuevamente vuelve a pensarse el estrecho de Magallanes como el paso interoceánico que permitiría el acceso a la especiería, reflotando los orígenes de la exploración magallánica. Por último, Valdivia propone la permanencia en el Estrecho al considerarlo no solo como puerto en el tránsito hacia la especiería, sino como parte integrante del reino de Chile. En otras palabras, el Estrecho se piensa como una fortaleza con el fin de que ningún adversario entre ni salga sin licencia ${ }^{68}$.

\section{CONCLUSIÓN}

El estrecho de Magallanes ha sido objeto de múltiples representaciones a lo largo de los primeros años de la conquista de América. El confín del mundo se instaló en el centro de la escena comercial entre España y las islas orientales, las cuales fueron objeto de proyecciones míticas signadas por el imaginario medieval y renacentista. Camino a las Molucas no solo estaba el Mar Océano, sino las costas del Nuevo Mundo que albergaban gigantes, caníbales y escenarios maravillosos. Esas variaciones interpretativas
65 Ibidem, p. 112.

66 Ibidem, p. 160.
67 Ibidem, p. 180.

68 Ibidem, p. 177. 
sobre el Estrecho dependieron, en gran medida, del interés depositado por aquella porción de tierra austral y del punto de vista de quienes emprendieron la tarea de llevar a la conciencia occidental un espacio que se erigía como una gran incógnita.

Los primeros navegantes que acompañaron a Fernando de Magallanes en la búsqueda de la tierra donde nacía la especiería, no tuvieron una imagen univoca del paso interoceánico; si bien es cierto que el mismo monarca consideró al Estrecho como un lugar de tránsito hacia el Maluco para consolidar su proyecto imperial, el entusiasmo demostrado por Antonio Pigafetta en su descripción del momento en que las naves alcanzaron finalmente el Mar del Sur implica una atención que exalta algunos puntos del trayecto como espacios en sí mismos. La diferencia estriba en la perspectiva que obtura o estimula, en el deseo de poseer y controlar aquellos páramos y, en definitiva, en la mirada del navegante que va más allá de las aguas que lo contienen y posa sus ojos en la potencialidad de aquel territorio. Sin embargo, el límite de aquella representación está en los alcances de la vista: a lo sumo el estrecho de Magallanes puede concebirse como un puerto que sirva para el paso de las embarcaciones hacia las islas Molucas. Nada se sabe de lo que existe tierra adentro y nada puede proyectarse sobre los indios que habitan en el lugar.

Hemos visto que la presencia de Pedro de Valdivia como emblema de la conquista y colonización del reino de Chile es un parteaguas en la historia del paso interoceánico ya que incorpora el estrecho de Magallanes en su propio proyecto territorial. En un primer momento, el Estrecho operará como frontera y límite que debe ser custodiado no solo por las fuerzas militares de la región, sino también por la venia legitimadora de la Corona que clarifique el poder del gobernador en un período de inestabilidad política que irriga la zona del Perú y alcanza al reino de Chile. Este será el momento en que el Estrecho dejará de significar un paso hacia la especiería, para convertirse en la vía de acceso de aquellos que intenten desestabilizar a Pedro de Valdivia. El objetivo es, entonces, cerrar ese paso y controlar la circulación; pero, sobre todo, explorar las costas para conocer aquello que se intenta dominar. El mapa costero hacia el Estrecho se delinea y el espacio vacío de representación se convierte en territorio de conquista.

Una vez consolidado el poder del extremeño, el paso interoceánico se re-semantiza, haciendo convivir el proyecto territorial del gobernador de Chile con el plan expansionista de la Corona: en primer lugar, porque se trata de un punto de conexión que estimula el flujo de personas y embarcaciones a escala planetaria facilitando el comercio; en segundo lugar, porque adquiere el carácter de frontera -ahora inofensiva debido al nombramiento de Valdivia- que motiva la colonización de un territorio no anexado; por último, porque deviene en referencia del proyecto territorial que Pedro de Valdivia lleva a cabo desde 1540: el objetivo será poblar y sustentar aquel paraíso que da comienzo a la grosedad de la gente con los recursos que emulan a las mejores ciudades españolas. En apenas treinta años, el estrecho de Magallanes pasa de ser una posta en el tránsito hacia la especiería a convertirse en un hogar potencial para los sufridos colonizadores del reino de Chile.

\section{BIBLIOGRAFÍA}

Anderson, P. (1974). Lineages of the Absolutist State. London-New York: Verso.

Beauchesne, K. (2013). Visión periférica. Marginalidad y colonialidad en las crónicas de América Latina (siglos XVI-XVII y XX-XXI). Madrid: Iberoamericana.

Correa Bello, S. (1986). Los fundamentos legales del primer nombramiento de Pedro de Valdivia. Historia, 21, 167-176.

De Certeau, M. (1996). La invención de lo cotidiano. Artes de hacer. México: Universidad Iberoamericana.

Ferreccio Podestá, M. (1991). El epistolario cronístico valdiviano y el scriptorium de conquista. En Cartas de don Pedro de Valdivia: que tratan del descubrimiento y conquista de la Nueva Extremadura. Edición de Miguel Rojas-Mix. Transcripción y notas de Mario Ferreccio Podestá. Barcelona: Lumen.

Giucci, G. (2014). Tierra del Fuego: la creación del fin del mundo. Buenos Aires: Fondo de Cultura Económica.

Goic, C. (2006). Letras del reino de Chile. Madrid: Iberoamericana Vervuert.

Greenblatt, S. (2008). Maravillosas posesiones. El asombro ante el Nuevo Mundo. Barcelona: Marbot ediciones. 
Gruzinski, S. (2010). Las cuatro partes del mundo. Historia de una mundialización. México: Fondo de Cultura Económica.

Guerin, M. (1992). El relato de viaje americano y la redefinición sociocultural de la ecúmene europea. Dispositio, 17(42-43), 1-19.

Invernizzi, L. (1984). La representación de la tierra de Chile en cinco textos de los siglos XVI y XVII. Revista Chilena de Literatura, 23, 5-37.

Invernizzi, L. (1990). 'Los trabajos de la guerra' y 'los trabajos del hambre': dos ejes del discurso narrativo de la Conquista de Chile (Valdivia, Vivar, Góngora Marmolejo). Revista Chilena de Literatura, 36, 7-15.

Lefebvre, H. (1974). The production of space. OxfordCambridge: Blackwell.

Lienhard, M. (1990). La voz y su huella: escritura y conflicto étnico-social en América Latina. La Habana: Casa de las Américas.

Long, P. (2015). Trading Zones in early Modern Europe. Isis, 106(4), 840-847.

Martinic, M. (1977). Historia del estrecho de Magallanes. Santiago: Editorial Andrés Bello.

Martinic, M. (2016). Una travesía memorable. Hallazgo y navegación del Estrecho de Magallanes (21 octubre-28 noviembre 1520). Punta Arenas: Talleres La Prensa Austral.

Massmann, S. (2013). Buscando camino por la mar: experiencia, geografía e imaginarios marítimos en relatos de navegación. Revista de Crítica Latinoamericana, 39(78), 209-232.

Onetto, M. (2017a). Modernidad, historicidad y construcción de territorialidades desde un pasaje-mundo. El estrecho de Magallanes tras su 'descubrimiento'. Magallania, 45(2), 37-58.

Onetto, M. (2017b). Reconsideraciones sobre la 'mala fama' de Chile durante el siglo XVI. Sophia Austral, 20, 5-29.
Pastor, B. (2008). El segundo descubrimiento. La conquista de América narrada por sus coetáneos (1492-1589). Buenos Aires: Edhasa.

Pratt, M. L. (2010). Ojos imperiales. Literatura de viajes y transculturación. Buenos Aires: Fondo de Cultura Económica.

Rabasa, J. (2009). De la invención de América. La historiografía española y la formación del eurocentrismo. México: Universidad Iberoamericana.

Raffestin, C. (2011). Por una geografía del poder. México: El Colegio de Michoacán.

Robles, H. (2008). The First Voyage around the World: From Pigafetta to García Márquez. Guaraguao, 12(27), 9-32.

Subirats, E. (1994). El continente vacío. La conquista del Nuevo Mundo y la conciencia moderna. México: Siglo XXI.

Trabulse, E. (1994). Ciencia y tecnología en el Nuevo Mundo. México: Fondo de Cultura Económica.

Triviños, G. (1996). El mito del tiempo de los héroes en Valdivia, Vivar y Ercilla. Revista Chilena de Literatura, 49, 5-26.

Vagnon, E. (2010). De la grèce antique au voyage de Magellan. Les modèles humanistes d'Antonio Pigafetta et de Maximilianus Transylvanus. Médiévales, 58, 99-111.

Vega, A. (2014). Los Andes y el territorio de Chile en el siglo XVI. Descripción, reconocimiento e invención. Santiago: Dirección de Bibliotecas, Archivos y Museos.

\section{FUENTES}

Medina, J. (1888-1896). Colección de Documentos Inéditos para la Historia de Chile. Primera serie, Tomos I, II, III, IV y VIII. Santiago: Imprenta Ercilla.

Valdivia, P. de. (1986). Cartas de relación de la conquista de Chile. Edición de Mario Ferreccio Podestá. Santiago: Editorial Universitaria. 